\title{
Best Practice Guidelines on Surgical Response in Disasters and Humanitarian Emergencies: Report of the 2011 Humanitarian Action Summit Working Group on Surgical Issues within the Humanitarian Space
}

\author{
Smita Chackungal, MD, MPH; ${ }^{1}$ Jason W. Nickerson, RRT, PhD(c) ${ }^{2}$ Lisa M. Knowlton, MD, MPH; ${ }^{3}$ \\ Lynn Black, MD, MPH; ${ }^{4}$ Frederick, M. Burkle Jr., MD, MPH, DTM $;{ }^{5}$ Kathleen Casey, MD; ${ }^{6}$ David Crandell; \\ Didier Demey; ${ }^{8}$ Lillian Di Giacomo, MD, MPH(c); ${ }^{9}$ Lena Dohlman, MD, MPH; ${ }^{10}$ Joshua Goldstein; ${ }^{11}$ \\ James E. Gosney Jr., MD, MPH; ${ }^{12}$ Keita Ikeda, PhD; ${ }^{13}$ Allison Linden, MD, MPH(c); ${ }^{14}$ \\ Catherine M. Mullaly, MD, MPH; ${ }^{15}$ Colleen O'Connell, MD; ${ }^{16}$ Anthony D. Redmond. MD; ${ }^{17}$ \\ Adam Richards, MD, MPH; ${ }^{18}$ Robert Rufsvold, MD $;{ }^{19}$ Ana L.R. Santos, MSc; ${ }^{20}$ Terri Skelton, MD $;{ }^{21}$ \\ Kelly McQueen, MD, $\mathrm{MPH}^{22}$
}

Division of General Surgery, University of Western Ontario, London, Ontario, Canada; Surgical Research Fellow Harvard Humanitarian Initiative, Harvard University, Cambridge, Massachusetts USA

Centre for Global Health, Institute of Population Health, University of Ottawa, Ottawa, Ontario, Canada Division of General Surgery, University of British Columbia Vancouver, British Columbia, Canada; Surgical Research Fellow, Harvard Humanitarian Initiative, Harvard University, Cambridge, Massachusetts USA

Department of Medicine, Massachusetts General Hospital, Boston, Massachusetts USA

tist, Harvard Humanitarian Initiative, Unersity, Cambridge, Massachusetts USA Director, Operation Giving Back, American College of Surgeons, Chicago, Illinois USA

Department of Physical Medicine and Rehabilitation, Harvard Medical School, Boston, Massachusetts USA Physical Therapist, Technical Advisor for Emergency and Rehabilitation, Handicap International, Brussels, Belgium John Hopkins Bloomberg School of Public Health, Baltimore, Maryland, USA; Department of General Surgery, University of California, Davis Medical Center, Sacramento, California USA

10. Assistant Professor, Harvard Medical School; Department of Anesthesia, Critical Care and Pain Medicine, Massachusetts General Hospital, Boston, Massachusetts USA

11. Principal Director for Economic Citizenship \& Disability Inclupion $\mathrm{Cec}$ for Finacil Inclusion at ACCION Inclusion, Center for Finacion Massachusetts USA

A

12. Handicap International, Takoma Park, Maryland USA Post Doctorate Fellow, Duke University Medical Center, Department of Anesthesia, Raleigh, North Carolina USA

4. Harvard School of Public Health, Boston, Massachusetts, USA; Georgetown University Medical Center, Washington, D.C. USA

15. Instructor, Harvard Medical School; Department of Anesthesia, Critical Care and Pain Medicine, Massachusetts General Hospital, Boston, Massachusetts USA Research Chief, Stan Cassidy Centre for Rehabilitation, Fredericton, New Brunswick, Canada

Humanitarian and Conflict Response Institute, University of Manchester, Manchester, UK

8. American Heart Association-Pharmaceutical Outcomes Rer Research Center, UCLA Department of Neurology, Los Anges, Califi, USA;

9. Medical Director, International Medical Corps Emergency Response, Libya

Response, Libya
Delft University of Technology, Delft, Netherlands

20. Delft University of Technology, Delft, Netherlands Department of Ant

Fellow, Harvard Humanitarian Initiative, Harvard University, Cambridge, Massachusetts; Valley Anesthesiology Consultants, Phoenix, Arizona USA

\section{Abstract}

The provision of surgery within humanitarian crises is complex, requiring coordination and cooperation among all stakeholders. During the 2011 Humanitarian Action Summit best practice guidelines were proposed to provide greater accountability and standardization in surgical humanitarian relief efforts. Surgical humanitarian relief planning should occur early and include team selection and preparation, appropriate disaster-specific anticipatory planning, needs assessment, and an awareness of local resources and limitations of cross-cultural project management. Accurate medical record keeping and timely follow-up is important for a transient surgical population. Integration with local health systems is essential and will help facilitate longer term surgical health system strengthening.

\section{Background}

The international humanitarian community often relies on best practice guidelines to ensure appropriate patient care in the field where circumstances are less than ideal. These guidelines inform and orient organizations and individuals who are providing care following major disasters, conflict and other humanitarian emergencies. The purpose of developing guidelines in this context is to encourage accountability and quality in the humanitarian response across the many levels of humanitarian agencies involved in relief efforts. Such guidelines help prepare responders in these settings as the challenges and dilemmas are often quite different than those encountered at home. The overarching goal is to effectively serve the population at risk while respecting their fundamental right to life with dignity. This goal reflects the philosophy underlying the Sphere Project's Humanitarian Charter, which acts as a general statement of principles that should govern all aspects of humanitarian activities. ${ }^{1}$

Professionalism has been evolving in the humanitarian community for more than a decade. Recent publications support not only humanitarian professionalism, but also a role for formal preparation and education through the establishment of core competencies. ${ }^{2,3}$ The 2010 Haitian earthquake uncovered deficiencies in and recommendations for best practices, standards, accountability and accreditation that must be addressed in a timely and accurate manner. ${ }^{4-6}$ The surgical community seeks to support and conform to those

\footnotetext{
Corresponding Author:

Smita Chackungal, MD, MPH

Division of General Surgery

University of Western Ontario

339 Windermere Road

London, Ontario Canada

N6A 5A5

E-mail: schackun@uwo.ca
}

ICRC = International Committee
of the Red Cross
$\mathrm{IO}=$ International organization
$\mathrm{NGO}=$ non-government
organization
$\mathrm{WHO}=$ World Health Organization

doi:10.1017/S1049023X12000064
Keywords: Surgical burden of disease; Surgical standards; Disaster medicine; Humanitarian

Abbreviations:

AS $=$ Humanitarian Action Summit (2011) 


\section{Surgical Issues Within the Humanitarian Space Working Group Summary}

Statement 1: Surgery and anesthesia are essential services in the crisis response, and along with rehabilitation, are part of the spectrum of care of the injured patient.

Statement 2: There should be minimum standards of core competencies and capabilities for providers of surgical care in crisis, to promote professionalization and standardization in the field.

Statement 3: Adequate planning for the surgical response in crisis should begin early, based on a needs assessment of the affected population.

Statement 4: There should be minimum standards of care in the following areas during planning of a surgical response in crisis:

\begin{tabular}{|l|l|l|}
\hline Program Planning & Equipment & Medications \\
\hline Security & Anesthesia Equipment & Anesthesia \\
\hline Electricity & Surgical and Rehab Equipment & Antibiotics and Prophylaxis \\
\hline Waste Management & Supplies & Pain Management \\
\hline Water & & Intravenous Fluids \\
\hline Sterilization & Services Provided & \\
\hline Blood Availability & & \\
\hline Personnel & & \\
\hline
\end{tabular}

Statement 5: There should be a surgical medical record for every patient consisting of:

Pre-operative Assessment

Consent

Operative Note/Anesthetic Record

Post-operative/Discharge Plan

Statement 6: There should be a minimum dataset to allow for standardized data collection for epidemiologic/field reporting of the surgical response in crisis.

Statement 7: Follow-up of the surgical patient should occur within an appropriate time frame, with the vierw towards long-term rehabilitation, prevention of disability, quality of life and community reintegration.

proposed standards. As such, a working group on surgical issues convened during the 2011 Humanitarian Action Summit (2011 HAS) to consider best practices.

Provision of surgery within the humanitarian context was first discussed during the 2009 Humanitarian Action Summit. The working group at that time developed the following recommendations focusing on three broad issues to improve the delivery of surgical care by humanitarian organizations: (1) anticipate and understand the local context and conduct a needs assessment; (2) incorporate best practices in the humanitarian delivery of surgery; and (3) incorporate data collection into humanitarian practice. ${ }^{7}$ The objective of the working group for 2011 HAS was to build on the work of the previous summit and further define guidelines for surgical and anesthesia provision by foreign teams and individuals in disasters and humanitarian emergencies. These guidelines will help facilitate coordination, cooperation, and collaboration; define scope of practice; and contribute to an ongoing dialogue on surgery and anesthesia best practices within the humanitarian community.

The goals and deliverables of the 2011 HAS working group were to:

1. Revisit the role of surgery in disasters, humanitarian crises, and conflict by reviewing the literature and developing consensus among experts in the field.
2. Define the role of international organizations (IO), nongovernmental organizations (NGO), and military-led surgical assets in providing surgical services and develop best practice guidelines for surgery and anesthesia in disasters, humanitarian crises, and conflicts.

\section{Working Group Preparation}

A detailed assessment of previous humanitarian surgical response to disasters and humanitarian emergencies was essential to develop guidelines and minimum standards that can be used to improve the outcomes of future humanitarian actions. With that in mind, a literature search was performed examining the role of surgery in disasters and other humanitarian emergencies. The goal of the literature review was to guide the working group's discussion of pre-departure planning for the provision of surgical care in crises. Information was gathered on patterns of morbidity and mortality, patterns of disease, resources required, and the outcomes of surgical interventions in crises to better understand health services delivery. During 2011 HAS, a group of experts in the fields of surgery, anesthesia, emergency medicine, rehabilitation medicine (physical and psychosocial), engineering, and humanitarian delivery were invited to discuss the specifics of best practices for surgical delivery in the humanitarian setting. Discussions held over the 


\begin{tabular}{|c|c|}
\hline Publication & Summary \\
\hline $\begin{array}{l}\text { Sphere Project Humanitarian Charter and } \\
\text { Minimum Standards in Disaster Response } \\
\text { (2011)[1] }\end{array}$ & $\begin{array}{l}\text { Not specific to surgical services or delivery } \\
\text { Broad guidelines and standards for the delivery of humanitarian relief }\end{array}$ \\
\hline $\begin{array}{l}\text { World Health Organization (WHO) Best } \\
\text { Practice Guidelines on Emergency Surgical } \\
\text { Care in Disaster Situations (2005)[8] }\end{array}$ & $\begin{array}{l}\text { Extracted from WHO manual "Surgical Care at the District Hospital"[9] } \\
\text { Describes basic clinical management of common surgical problems encountered in } \\
\text { disaster } \\
\text { - Resuscitation } \\
\text { - Wound management } \\
\text { - Fractures } \\
\text { - Amputations } \\
\text { Guidelines included for proper antibiotic usage, post-operative care and anaesthesia }\end{array}$ \\
\hline $\begin{array}{l}\text { International Committee of the Red Cross } \\
\text { (ICRC) War surgery Guidelines (2009) [10] }\end{array}$ & $\begin{array}{l}\text { ICRC publishes basic reference manual for surgeons embarking on humanitarian } \\
\text { missions in settings of conflict since } 1988 . \\
\text { Comprehensive handbook on characteristics and mechanisms of war injury, } \\
\text { epidemiology, triage, wound scoring, and clinical management of war related injuries. } \\
\text { Guidelines on antibiotic use, principles of surgical management, and blood } \\
\text { transfusions in resource limited settings. }\end{array}$ \\
\hline $\begin{array}{l}\text { WHO/PAHO Guidelines on International } \\
\text { Foreign Medical Teams (2010) [3] }\end{array}$ & $\begin{array}{l}\text { Minimum standards for medical teams and facilities after sudden impact disasters, } \\
\text { focusing on health services provided rather than type of field hospital to be mobilized } \\
\text { International registration as a first step to eventual accreditation of foreign medical } \\
\text { teams }\end{array}$ \\
\hline $\begin{array}{l}\text { World Federation of Societies of } \\
\text { Anesthesiologists (WFSA) International } \\
\text { Standards for the Safe Practice of } \\
\text { Anesthesia (2008)[11] }\end{array}$ & $\begin{array}{l}\text { Best practices and standards for anesthesia delivery in all environments } \\
\text { Not specific to disaster or humanitarian crises }\end{array}$ \\
\hline $\begin{array}{l}\text { WHO Guide to Anesthetic Infrastructure and } \\
\text { Supplies at Various Levels of Health Care } \\
\text { Facilities (2006)[12] }\end{array}$ & $\begin{array}{l}\text { Extracted from WHO manual "Surgical Care at the District Hospital" [9] } \\
\text { Details the various procedures and anesthesia training required at different levels } \\
\text { of health facilities, and the essential medications and equipment appropriate } \\
\text { at each level } \\
\text { Not specific to disaster or humanitarian crises }\end{array}$ \\
\hline
\end{tabular}

Table1 - Existing guidelines applicable to humanitarian delivery of anesthesia or surgical care

course of the summit addressed discrepancies between existing guidelines and their applicability to disaster and conflict settings. The aim was to translate these broad guidelines into minimum practice standards for humanitarian surgery, anesthesia and rehabilitation delivery that can be effectively implemented on the ground by any IO/NGO.

\section{Working Group Findings}

Existing guidelines relevant to humanitarian delivery and anesthesia/surgical care in disasters or conflicts were identified by the working group and are summarized in Table 1. These guidelines, in addition to other applicable resources from published literature provided the framework for the working group discussions on best practices for foreign surgical teams. Recommendations were supported by evidence where it existed, adapted from existing guidelines, and from expert opinions of working group members with experience in crisis settings.

Over the course of the HAS, a consensus opinion was achieved regarding the importance of the following fundamental themes in planning surgical relief efforts:

- The importance of surgical, anesthesia and rehabilitation services in the crisis response
- The need for ongoing professional development of foreign medical teams

- The importance of disaster-specific anticipation that includes probable injuries and illnesses, and a knowledgebase of local capacity and indigenous resources

- Team member composition and pre-deployment preparation with an emphasis on providing services in austere settings with cultural competence

- Coordination with local health professionals and health systems

- Pre-departure planning for equipment, medications, range of services/mandate

- Medical record keeping including the importance of informed consent, post-operative and discharge planning

- Data tracking/epidemiologic reporting

- The need for coordinated post-operative follow-up care including the provision of rehabilitation services.

\section{1) The importance of surgery and anesthesia in disaster relief and preparedness}

Providing early surgical care is crucial to an effective response in humanitarian emergencies. Appropriate surgical interventions, obstetrical care and anesthesia services should be identified as 
Best Practice Guidelines on Surgical Response in Disasters and Humanitarian Emergencies

essential health services within disaster relief efforts. The spectrum of care should include preoperative planning, evaluation and resuscitation, anesthesia and pain management, and post-operative care including the coordination of rehabilitation services.

\section{2) Minimum standards for capabilities and competencies}

There should be a set of minimum standards for capabilities and competencies required of international organizations and individuals providing surgical care within disaster and conflict settings. ${ }^{14}$ Such standards provide the basis for the development of national or international registries of multidisciplinary surgical provider teams. These registries will improve accountability, allow for appropriate training and accreditation, and help reinforce core competencies needed for an early coordinated humanitarian response. ${ }^{4-5,15}$ They also serve to promote the professionalization of disaster relief providers with the intention of avoiding the deployment of poorly prepared teams to these complex environments.

\section{3) The multidisciplinary surgical response and adequate pre- deployment preparation}

Planning for a multidisciplinary surgical response and reception of these teams in disasters and conflicts must begin early. It must begin with planning based on disaster-specific injury and endemic illness anticipation, knowledge of local capacities, be inclusive of the team's logistical and professional needs, and be aligned with the team's determined mandate and range of provided services. An assessment of the team infrastructure and capacity must also include the resources for anesthesia and pain management, surgical capabilities, and the ability to provide peri-operative care, rehabilitation and psychosocial services. A basic understanding of the local culture and its relationship to traditional medical practices must be considered. The team mandate should be based on a needs assessment of the crisisaffected population and should be reflective of the team's capacity, experience and capabilities. ${ }^{16}$

\section{4) Minimum standards required for program planning, equip- ment, medications, and range of services provided}

The following minimum standards of care provide a guideline for teams and individuals in setting up a surgical program that is self-sufficient, flexible and adaptable to the needs of the local environment and context. Prior to beginning surgical clinical care, the necessary building blocks must be in place to ensure that treatment is provided safely and competently. Challenges must be anticipated and contingency plans should exist. It is important that early liaison with local systems occur. There should be open communication with other surgical and rehabilitative relief efforts including local teams for collaboration and coordination of services. A rapid needs assessment of the affected population should occur early, prior to surgery being performed. ${ }^{16,17}$ Without these minimum standards being met, surgical interventions should not begin in disaster, humanitarian crisis, or conflict settings.

\section{Program Planning}

\section{Security}

As a minimum standard of care in disaster settings and humanitarian emergencies, there needs to be an appropriate organizational-level response to an insecure environment including situational awareness, contingency plans, evacuation capabilities and adequate resourcing. Teams should consider the clinical services impact of having to evacuate staff and patients during a worsening security situation and should have an unambiguous plan for managing unsafe situations.

\section{Electricity}

As a minimum standard of care, individuals and teams need to be self-sufficient in meeting the energy demands required for delivery of surgical care. This includes, but is not limited, to generators, fuel, batteries, disaster-tested portable solar devices, or other means of meeting electricity needs for equipment and personal use. An energy policy should be elaborated to estimate and optimize the energy demand from procedure-specific devices and peripheral equipment. Dependence on the national power grid of the country should not be assumed given the constantly evolving environment typical of humanitarian crises.

\section{Waste and Sanitation}

As a minimum standard of care in disaster settings and humanitarian emergencies, there needs to be a safe and effective means of waste management including a safe method of disposing of sharps and hazardous biological waste. The World Health Organization (WHO) guidelines on the management of health care associated waste should be followed. ${ }^{18}$

\section{Water}

Existing Sphere guidelines on water required for the hospitalized patient are 40-60 L/day. ${ }^{1}$ This standard does not address the specific water requirements for the peri-operative timeframe. Médecins sans Frontières(MSF) guidelines for water usage suggest 100-300 L/patient/surgical procedure. ${ }^{7}$ As a minimum standard of care in disaster settings and humanitarian emergencies, it is recommended that teams plan for adequate water resources that exceed Sphere published minimums if they undertake surgical activities. Sufficient water should be stored on the premises to ensure a steady and uninterrupted supply.

\section{Sterilization}

As a minimum standard of care in disaster settings and humanitarian emergencies, the sterilization method should be able to accommodate the variety and size of surgical instruments and equipment needed in the field. The WHO recommends autoclaving or steam sterilization with prior disinfection in a chlorine solution as the primary method. ${ }^{8}$ Treatment with chemical antiseptics such as soaking in formaldehyde or chlorhexidine should only be used for sharp instruments, catheters and tubes. ${ }^{8}$ Simple boiling is unreliable and should be avoided. ${ }^{9}$

\section{Blood Availability}

A living donor blood bank and the capability for ABO typing and screening for infectious diseases should be the minimum requirement for all operative procedures with more than a minimal risk of hemorrhage. The $\mathrm{WHO}$ recommends that minimum screening include Human Immunodeficiency Virus (HIV), Hepatitis B, Hepatitis C, and syphilis. ${ }^{19}$ Additional screening for transfusion transmitted infections is necessary in most tropical settings. Screening protocols should be tailored to local epidemiology such as for Chagas disease or malaria in endemic areas. ${ }^{19}$ When anonymous testing of donor blood is not feasible, voluntary counselling and testing services (e.g., for HIV) should 
be provided. The aim of transfusion is to save a life and prevent morbidity. When an effective blood cold chain does not exist, the transfusion of fresh, whole blood is preferred. ${ }^{20}$ Although immunologic transfusion reactions and anaphylaxis are rare, clinicians should be prepared to recognize and treat these conditions. The International Committee of the Red Cross (ICRC) recommends that transfusion should be limited in cases of life-threatening massive hemorrhage, coagulopathy or shock. ${ }^{10}$ Although transfusion protocols exist for military $21-23$ and civilian 24,25 use in humanitarian settings, additional work is needed to evaluate the safety and efficacy of these standard protocols in crisis. Unnecessary transfusions are a waste of a scarce resource, and development of disaster-specific transfusion criteria is encouraged.

\section{Personnel}

Teams should be composed of professionals who are experienced in triage, anesthesia, rehabilitation, and adult and pediatric general surgical, orthopedic and obstetric procedures. Core teams should be supplemented with other specialists if there is sufficient need based on assessments of population needs including available resources and gaps in service delivery. Auxiliary staff should include logisticians, translators, and health service managers. Utilizing and encouraging leadership among local health workers and integrating with local health services must be practiced at all levels whenever possible, to facilitate health system transition and recovery beyond the acute stages of the crisis.

\section{Equipment}

As a minimum standard of care in disaster settings and humanitarian emergencies, all equipment should be appropriate to the local environment and context, and suitable for transportation. Technology should be consistent with what is available locally and with what can be supported on the ground and sustained by local services. Members of the team must be familiar with the equipment, and have a plan in place to deal with equipment failure or malfunction. Donated equipment must be in working order and suitable to the local context, considering the need for repairs and preventative maintenance. The WHO generic essential emergency equipment list should be used as a guide. ${ }^{26}$

\section{Oxygen Availability}

Oxygen should be readily available for all surgical procedures, provided, at a minimum, by an oxygen concentrator. The World Federation of Societies of Anesthesiologists (WFSA) highly recommends the availability of supplemental oxygen. ${ }^{11}$

\section{Anesthesia Equipment}

As a minimum standard of care in disaster settings and humanitarian emergencies, a pulse oximeter and a non-invasive blood pressure cuff should be available. Pulse oximetry has been shown to be a simple technology that can prevent anesthesia-related complications. ${ }^{27} \mathrm{~A}$ precordial stethoscope or equivalent may be beneficial in these environments depending on familiarity and experience with the equipment. The WHO recommends additional equipment such as bag valve masks, laryngoscopes, oral airways, endotracheal tubes and cricothyrotomy kits. 8,12 Anesthesia should be provided by a dedicated person with appropriate training who is responsible for the care and monitoring of the patient during every surgical procedure. ${ }^{28}$
Surgical and Rehabilitation Equipment

Surgical equipment sufficient to perform the range of surgical procedures expected according to the disaster/conflict is required at a minimum, bearing in mind that technical failures might occur. This should include equipment necessary for emergency obstetric surgery, trauma surgery, simple and complex wound care, and non-crisis related procedures (i.e., hernia repair, bowel obstruction, obstetrics). ${ }^{29-31}$ Surgical teams that provide postoperative physical rehabilitation should coordinate with physical rehabilitation service providers for provision of required assistive devices (e.g., prostheses) and mobility aids (e.g., wheelchairs and crutches) as necessary. ${ }^{1}$

\section{Supplies}

Personal protective equipment such as sterile gloves, gowns, masks, and eye protection are essential items and there should be a sufficient amount appropriate for the duration of the relief effort. Universal precautions should be maintained at all times. The use of blunt suture needles and other safety devices should be used to minimize percutaneous sharps injuries in and out of the operating room. ${ }^{32}$ In order to maintain this standard of care in humanitarian settings, it may be necessary to conduct brief trainings prior to departure or in the field to familiarize foreign and local staff with available safety devices and protocols. There should also be a sufficient amount of disposable sterile supplies available. The rapid needs assessment should include the establishment of an alternative local supply chain for basic consumables that does not compromise local resources. Waste should be minimized to avoid undue contamination of the environment.

\section{Medications}

In the absence of evidence-based guidelines specific to disaster or conflict situations, refer to the World Health Organization's (WHO) Model List of Essential Medicines. ${ }^{33}$ The WHO Model List is globally recognized and forms the basis of many national formularies, allowing for essential medications to be available in-country. Contextually-specific medication lists are recommended. Any augmentation to these recommendations should be considered with regards to continued sustainability and procurement means within the country and existing formularies as applicable. Expired drugs should not be brought to disaster sites by humanitarian relief teams.

\section{Anesthesia}

As a minimum standard of care in disaster settings and humanitarian emergencies, anesthesia drugs as recommended by the WHO Model List of Essential Medicines should be used. Drugs and equipment for regional peripheral and neuraxial anesthesia, procedural sedation, local anesthesia as well as general anesthesia should be included. Resuscitative drugs must be available and sedatives, opioids, benzodiazepines, non-steroidal anti-inflammatories and ketamine should be considered. ${ }^{34,35}$ Drugs that have the potential for diversion should be kept in a locked and safe area until needed by qualified personnel.

\section{Antibiotics and Prophylaxis}

The WHO Model List of Essential Medicines should form the minimum standard for medications in crisis settings, with additions made as warranted and appropriate. Drugs that can appropriately cover the typical organisms responsible for surgical infections should be included. The ICRC antibiotic protocol 
Best Practice Guidelines on Surgical Response in Disasters and Humanitarian Emergencies

for surgical procedures provides an excellent background for such circumstances. ${ }^{10}$ Knowledge of endemic infectious and non-communicable diseases is essential for both pre- and postoperative care and rehabilitation. Tetanus prophylaxis should be available and given where appropriate. ${ }^{8}$ Prophylaxis for endemic diseases should be available for clinical staff as well as patients. Staff should be up-to-date with vaccinations for endemic diseases, as well as those transmitted percutaneously such as Hepatitis B. Post-exposure HIV prophylaxis for needle stick injuries should be available.

\section{Pain Management}

The members of the working group reaffirm that access to pain treatment is a fundamental human right. ${ }^{36,37}$ Effective peri-operative pain management is an essential component of the treatment of the surgical patient. Inadequate pain control is both inhumane and may result in increased morbidity or mortality. ${ }^{37}$ Effective pain management improves patient comfort and satisfaction, allows for earlier mobilization with fewer pulmonary and cardiac complications, faster recovery, earlier reintegration into the community, and a reduction in the development of neuropathic pain. Good analgesia is generally possible even in low resource settings, and preparation to provide this care must be made along with all other disaster response preparations. ${ }^{38}$ As a minimum standard of care in disaster and humanitarian emergencies, all modalities for pain management must be considered (pharmacologic and nonpharmacologic), and appropriate pre-operative assessment and post-operative monitoring is essential. A WHO style analgesia 'pain ladder' should be employed whenever possible. ${ }^{39}$ Pain assessment should be accomplished using a universal, standardized tool which is appropriate and acceptable to the local populace. A non-verbal tool, such as a visual analogue scale, should be considered.

\section{Fluids}

As a minimum standard of care in disaster settings and humanitarian emergencies, sterile, peri-operative intravenous fluids must be available. There needs to be a plan in place for a stable supply chain to obtain intravenous fluids, recognizing that the amount and type that can be transported initially may be limited.

\section{Range of services expected to be provided}

As a minimum standard of care in disaster settings and humanitarian emergencies any operation or procedure performed should take into consideration the capabilities and limitations to providing effective and safe post-operative care and rehabilitation. Existing evidence suggests that foreign medical teams and foreign field hospitals are likely to encounter a range of surgical pathologies both directly and indirectly related to crises. ${ }^{29}$ Complex wound care, infections, fracture care, and amputations are expected to be prominent in the aftermath of certain disasters. During the initial stages of a response, unless specifically requested, single-service surgical teams ought to be discouraged in favour of surgical teams capable of a broad array of procedures (general surgery, paediatric surgery, orthopedics, plastic surgery, obstetrics \& gynecology). Surgical teams should be capable of managing the acute surgical needs of patients affected by crises, as well as the pre-existing burden of surgical disease and associated need for rehabilitation; this combined requirement may exceed the capabilities of existing health facilities and resources. Prioritization of surgical procedures should follow what has been previously recommended in the developing world. ${ }^{31}$ Rehabilitation planning begins from day one in the care of victims.

\section{5) Surgical medical record keeping including informed consent}

Patient registration and documentation of all subsequent clinical activities is essential to the optimum management of the patient and should have the capability to integrate with host country local health information systems to build future capacity and coordination. Medical records should travel with the patient and should be interpretable and translatable between service providers, both during and beyond the crisis. As a minimum standard of care in disaster settings and humanitarian emergencies, a medical record must exist for every surgical patient.

Minimum standards for the surgical medical record The surgical medical record should consist of the following: Preoperative Assessment, Consent, Operative/Anesthetic Note, Post-Operative/Discharge Plan. These items form the core of the surgical medical record and should be in addition to any other hospital record the patient may have.

\section{Preoperative Assessment}

A preoperative evaluation should be undertaken on every individual planned for surgery to assess anesthetic and peri-operative risk ${ }^{40}$, as well as the anticipated need for rehabilitative care.

\section{Consent:}

An important element of patient care is obtaining informed consent for surgery. This must include a discussion weighing the risks and benefits of intervention against conservative management, as well as potential surgical and anesthesia complications involved in the procedure. Surgical and anesthesia care providers have an ethical obligation to appropriately obtain informed consent from patients undergoing operative procedures, and patients have a right to pertinent information concerning their condition and treatment options. This idea of consent reflects the value of respecting a patient's right to autonomy and self-determination as enshrined in the United Nations Declaration of Human Rights, ${ }^{41}$ and should not be neglected, even in times of crisis. All attempts should be made to seek consent from the patient, family or culturally appropriate designate. Explanations should be clear and in the patient's own language. The use of translators in this setting is important. Regardless of how the consent is obtained, a record of the consent should be included in the medical record. A separate consent should be obtained for photo or video recordings of the patient, recognizing a patient's right to privacy.

\section{Operative Note and Anesthetic Record}

An operative note should be recorded with every procedure in the medical record and at a minimum include: patient identity, age, sex, preoperative diagnosis, postoperative diagnosis, procedure, surgeon, anesthetic technique, blood loss estimate, transfusion requirement, intra-operative findings, complications, post-operative condition, and post-operative plan. An anaesthetic record should be completed for every procedure, and at a minimum should include: interval recordings of oxygen saturation, heart rate, respiratory rate and blood pressure; length and type of anesthetic; amount and type of drugs or fluids administered; and length of the procedure. ${ }^{28,40}$ The use of a patient safety checklist in every operative setting is supported and should be standard of care. ${ }^{42}$ 
Post-operative and Discharge plan

It is critical to recognize that there are often multiple diverse medi$\mathrm{cal}$ and surgical teams rapidly rotating during a crisis. Consequently, clear and specific instructions should accompany every post-operative patient. As a minimum standard of care, every individual that has undergone an operation should have a both a post-operative and discharge plan in place that is communicated to the ongoing treatment team and receiving provider(s), the patient and the primary caregiver. These plans should include instructions for ongoing care and follow-up, possible complications to be aware of and both the medical and functional goals of treatment and recovery.

\section{6) Minimum dataset for epidemiologic reporting of pathology, caseload and patient demographics, surgical outcomes and quality indicators}

Minimum datasets and reporting guidelines for publication of surgical field reports during disasters and emergencies are needed to improve the quality of published reports. As a minimum standard of care in disasters and humanitarian emergencies, there should be standardised reporting for epidemiologic investigation and sharing of information. There should be further examination of an operationally useful information system for prospective and retrospective analysis by examining the experience of other countries and organizations. A good model to follow is the ICRC wound database and registry which has been in existence since 1990. This database records detailed information on wounds including time since injury, mechanism of injury, anatomic site of injury, wound score, major operation performed, and relevant clinical and administrative data. ${ }^{10}$

\section{7) Minimum standards of post-operative follow-up care}

As a minimum standard of care in disaster settings and humanitarian emergencies every individual undergoing a surgical procedure must be seen for follow-up within an appropriate time frame by the treating team or designated care provider. It is the responsibility of the surgical treatment team to ensure this occurs. Community-based follow up treatment plans should be developed that support local health system capacity building. Emergent and longer-term follow-up planning should always be organized in concert with local health systems. Capacity of local health care systems must be understood and supported where necessary. Working with local providers and services from the beginning promotes continuity of care. In humanitarian emergencies, the issue of follow-up becomes more complex given the often transient nature of the population served. Missed injuries and ongoing wound care problems are some of the potential complications that have been demonstrated in a previous follow-up study of earthquake victims. ${ }^{43} \mathrm{It}$ is possible for surgical teams to provide quality follow-up care in resource constrained settings. Operation Smile, a surgical NGO that provides cleft-lip surgery is one example. ${ }^{45} \mathrm{~A}$ portable medical record system that can be used by a range of clinicians in different settings becomes essential in these situations.

\section{Conclusion}

Humanitarian crises are events that overwhelm a regional authority's capacity to meet the needs of the affected population in core vital areas, including basic social and health services. The disruption of already weakened health systems tends to unmask pre-existing unmet health needs of the population. This includes existing healthcare worker shortages which negatively impacts surgical, anesthesia, and rehabilitation care bringing the burden of surgical disease to a more severe crisis level in many areas of the world. It is in this setting where the international community's assistance is most required. The recent crises in Haiti and Japan highlight the importance of preparedness in mitigating the effects of natural disasters and the value of planning for large scale humanitarian relief efforts. Given the crucial role of surgical care in the aftermath of disasters, surgical and surgical rehabilitation services must be viewed as essential health services along with other public health priorities such as shelter, food, clean water and sanitation.

The normal high level of coordination involved in surgical care delivery is complicated by the realities of modern humanitarian emergencies, which involve an increasing number of actors providing humanitarian relief. The inclusion of care for the injured patient as an essential health service in the most recent (2011) Sphere Handbook not only underscores the value of trauma, surgical services and post-operative rehabilitation for trauma-related injury in humanitarian activities, but also the importance of properly prepared and adequately trained surgical care providers. ${ }^{1}$ Novel proposals such as an Emergency Surgical Coalition could serve as a potential solution to the organizational challenges of multi-agency surgical provision during humanitarian emergencies. ${ }^{45}$ The recognition that surgery is a multidisciplinary and multifaceted endeavour, especially in austere environments, reveals a need for greater coordination and standardization among all players as well as the necessity of engaging and empowering local health authorities. The development of surgical best practices within the humanitarian space will ensure accountability and quality of services for the delivery of surgical, anaesthesia and rehabilitative care.

How best to transition from short-term emergency surgical relief efforts to longer term health systems strengthening remains a key challenge for the global surgical community. Proper team planning, the provision of context appropriate medication and equipment, comprehensive surgical medical record keeping, surgical outcome reporting, and timely and appropriate postoperative follow-up are all important components that must be considered the minimum standard of care for any individual or group who will provide surgical services in disaster settings and humanitarian emergencies. It is the intention of this working group to launch an ongoing dialogue that will eventually move the surgical community closer to the evidence base required for robust best practice guidelines to better prepare a future generation of surgical humanitarians.

References

1. Sphere Project: Sphere Handbook: Humanitarian Charter and Minimum Standards in Disaster Response. 2011.Available atwww.sphereproject.org/content/view/682/32/ lang,english/. Accessed 14 April 2011.

2. Keane M, Pack ME, Greenough PG, Burkle FM Jr: The professionalization of humanitarian health assistance: report of a survey on what humanitarian health workers tell us.PrehospDisast Med.2009;24(4):s204-6.

3. Bradt DA, Drummond CM: Professionalization of Disaster Medicine-an appraisal of criterion-referenced qualifications. PrehospDisast Med. 2007;22(5):360-8.

4. World Health Organization/Pan American Health Organization: Guidelines on International Foreign Medical Teams. Proceedings of the WHO/PAHO technical consultation on International Foreign Medical Teams (FMTs) Post Sudden Onset Disasters (SODs). Havana, Cuba. 7-9 December 2010.

5. Working Groups Background Paper: Accountability, Quality Control and Reporting. Proceedings of the WHO/PAHO Technical Consultation on International Foreign Medical Teams (FMTs) Post Sudden Onset Disasters (SODs). Havana, Cuba. December 7-9, 2010. 
6. Working Groups Background Paper: Registration, Certification and Coordination. Proceedings of the WHO/PAHO Technical Consultation on International Foreign Medical Teams (FMTs) Post Sudden Onset Disasters (SODs). Havana, Cuba. December 7-9, 2010.

7. McQueen K, Parmar P, Keane M, Broaddus S et. al: Burden of Surgical Disease: Strategies to manage an existing public health emergency. PrehospDisast Med. 2009;24(4):s228-31.

8. World Health Organization: WHO Best Practice Guidelines on Emergency Surgical Care in Disaster Settings. 2005. Available at www.who.int/surgery/publications/ disastermanagguide.pdf. Accessed 14 March 2011.

9. World Health Organization: Surgical Care at the District Hospital. Geneva: WHO, 2003.

10. Gianou C, Balden M, (eds). War SurgeryVol 1. Geneva: International Committee of the Red Cross, 2009.

11. World Federation of Societies of Anesthesiologists: 2008International Standards for the Safe Practice of Anesthesia.2008. Available at www.anaesthesiologists. org/guidelines/practice/2008-international-standards-for-a-safe-practice-ofanaesthesia. Accessed 04 April 2011.

12. World Health Organization: Guide to Anesthetic Infrastructure and Supplies at Various Levels of Health Care Facilities.2006. Available at www.who.int/surgery/ publications/GuideAnestheticInfrastFormatted06.pdf. Accessed 04 April 2011.

13. American Society of Anesthesiologists: Standard Guidelines and Statements. Availableatwww.asahq.org/For-Healthcare-Professionals/Standards-Guidelinesand-Statements.aspx. Accessed 19 April 2011.

14. Seynaeve G, Archer F, Fischer J, Lueger-Schuster B et. al: International standards and guidelines on education and training for the multi-disciplinary health response to major events that threaten the health status of a community. PrehospDisast Med.2004;19(3):186-7.

15. Redmond AD, O'Dempsey TJ, Taithe, B: Disasters and a register for foreign medical teams. Lancet. 2011;377:1054-1055.

16. Redmond AD: ABC of conflict and disaster: Needs assessment of Humanitarian Crises. BMJ. 2005;330:1320-22.

17. International Committee of the Red Cross, Guidelines for emergency assessment. Geneva: ICRC, 2005.

18. Pruss A, Giroult E, Rushbrook P, (eds). Safe Management of Wastes from Health Care Activities. Geneva: WHO, 1999.

19. World Health Organization: Screening donated blood for transfusion transmittable infections. 2010. Available at www.who.int/bloodsafety/Screening DonatedBlood forTransfusion.pdf. Accessed 31 March 2011.

20. World Health Organization:Manual on management, maintenance and use of blood cold chain equipment. Geneva: WHO, 2005.

21. Grosso SM, Keenan JO: Whole blood transfusion for exsanguinating coagulopathy in a US field surgical hospital in postwar Kosovo. J Trauma. 2000;49(1):145-148.

22. Hrezo RJ, Clark J: The walking blood bank: an alternative blood supply in military masss casualties. Disaster Manag Response. 2003;1(1):19-22.

23. Malsby R, 3rd, Frizzi J, Ray P, Raff J: Walking donor transfusion in a far forward environment. South Med J. 2005;98(8):809-810.

24. Raytheon Polar Services Company: Blood Bank/Emergency Transfusion. 2006 Availableatwww.nsf.gov/about/contracting/rfqs/support_ant/docs/environ_health_ safety/usap_saddh_policy_man/bloodbankemergtransfusions-305.pdf.Accessed 15 April 2011.
25. Laouabdia-Sellami K, Dubourg D, Gillet P, Marcelis L, Rosseel P, I S. Blood transfusion in remote areas: Médecins Sans Frontières 1999.

26. World Health Organization: Generic Essential Emergency Equipment List. 2006. Availableatwww.who.int/surgery/publications/EEEGenericListFormatted\%2006. pdf. Accessed 04 April 2011.

27. Møller JT, Johannessen NW, Espersen K, Ravio O: Randomized evaluation of pulse oximetry in 20,802 patients:II. Perioperative events and postoperative complications. Anesthesiology 1993;78 445-53.

28. American Society of Anesthesiologists: Basic Anesthetic Monitoring Standards. 2011. Available atwww.asahq.org/For-Healthcare-Professionals/StandardsGuidelines-and-Statements.aspx. Accessed 19 April 2011.

29. Nickerson JW. Surgical Care in Crises: What do the Data tell us? Presentation at Humanitarian Action Summit 2011, Cambridge, Massachusetts, 05 March 2011.

30. Chu K, Rosseel P, Trelles M, Gielis P: Surgeons without borders: A brief history of surgery at Médecins sans Frontières. World Journal of Surgery. 2010:34(3): 411-414.

31. Mock C, Cherian M, Julliard C, Donkor P, Bickler S, Jamison D, McQueen K: Developing Priorities for addressing Surgical Conditions globally: Furthering the link between surgery and public health policy. World Journal of Surgery. 2010;34:381-385.

32. American College of Surgeons: Statement on Sharps Safety. 2007. Available at www.facs.org/fellows_info/statements/st-58.html. Accessed 20 April 2011.

33. World Health Organization: WHO Model List, 16th ed (updated). 2010. Available at www.who.int/medicines/publications/essesntialmedicines/en/index.html. Accessed 14 Mar 2011.

34. Schnittger T: Regional anesthesia in Developing Countries. Anesthesia. 2007;62(Suppl.1):42-47.

35. Craven R: Ketamine. Anesthesia.2007;62(Suppl 1):48-53.

36. Lohman D, Schleifer R, Amon JJ. Access to Pain Treatment as a Human Right. BMC Med. 2010;8:8

37. Brennen F, Carr D.B, Cousins M: Pain Management: A Fundamental Human Right. Anesthesia and Analgesia. 2007;105(1):205-221.

38. Size M, Soyannwo OA, Justins DM: Pain Management in Developing Countries. Anesthesia. 2007;63(Suppl 1):38-43

39. Charlton E: The management of post operative pain. Update in Anaesthesia. 1997;7:1-7.

40. American Society of Anesthesiologists: Statement of Documentation of Anesthesia Care. 2008. Available atwww.asahq.org/For-Healthcare-Professionals/StandardsGuidelines-and-Statements.aspx. Accessed 19 April 2011.

41. United Nations: Universal Declaration of Human Rights. 2007. Available at www. un.org/en/documents/udhr/index.shtml. Accessed 14 April 2011.

42. Haynes AB, Weiser TG, Berry WR et. al: A Surgical Safety Checklist to reduce Morbidity and Mortality in a Global Population.N EngJ Med. 2009;360:491-9.

43. Roy N, Shah H, Patel V, Bagalkote H: Surgical and psychosocial outcomes in the rural injured - a follow-up study of the 2001 earthquake victims. Injury. 2005;36(8):927-34.

44. Bermudez L, Carter V, Magee W Jr, Sherman R, Ayala R: Surgical Outcomes auditing systems in Humanitarian Organizations. World Journal of Surgery. 2010;34(3):403-10.

45. Chu K, Stokes S, Trelles M, Ford N: Improving Effective Surgical Delivery in Humanitarian Disasters: Lessons from Haiti. PLoS Med. 2011;8(4):e1001025. 